\title{
UM ESTUDO SOBRE A OPERAÇÃO DA DIVISÃO DOS NÚMEROS CONTIDA NO TRAITÉ DE GERBERT NA VERSÃO DE 1843
}

\section{A STUDY ON THE OPERATION OF THE NUMBER DIVISION CONTAINED IN THE GERBERT TRAITÉ IN THE 1843 VERSION}

\author{
Suzî̂ Maria de Albuquerque ${ }^{1}$ \\ Instituto Federa de Educação, Ciência e Tecnologia do Ceará
}

\begin{abstract}
Resumo
Este estudo é um desdobramento da pesquisa de mestrado realizada na perspectiva da construção de uma interface entre a história e o ensino, que versou sobre o uso do ábaco de Gerbert para os casos da operação da multiplicação dos números aplicada na formação de professores de matemática. Considerando-se que o Traité de Gerbert na versão de 1843 contém, além da operação mencionada, a divisão dos números, elencouse neste trabalho o objetivo de conhecer os princípios desta última operação nos casos contidos no referido texto. Para tanto, realizou-se um estudo documental buscando informações no passado, advindas do texto selecionado e de outros escritos do mesmo período que revelaram como os praticantes de matemáticas do século X lidavam com a divisão dos números, sobretudo no uso do ábaco de Gerbert. Nesse sentido, foi realizada uma análise preliminar do contexto histórico mencionado em três esferas: a contextual, a historiográfica e a epistemológica. A partir disso, foi possível compreender que a divisão dos números no uso do ábaco de Gerbert se dá pela subtração, obtendo-se o quociente da ideia de quantas vezes o dividendo pode conter o divisor. Além disso, traz a divisão pelo método das diferenças, no qual a operação é realizada se dividindo por um divisor auxiliar que facilitaria a obtenção dos resultados no sistema do ábaco. Apesar de este ser um estudo inicial, é possível indicar elementos que podem colaborar na compreensão da operação da divisão por meio de seu algoritmo moderno. Entretanto, este último aspecto será aprofundado em estudos futuros.
\end{abstract}

Palavras-chave: Divisão dos números. Ábaco de Gerbert. Interface entre história e ensino.

\begin{abstract}
This study is an unfolding of the master's research carried out in the perspective of building an interface between history and teaching, which dealt with the use of Gerbert's abacus for the cases of the operation of the multiplication of numbers applied in the training of mathematics teachers. Considering that Gerbert's Traite in the 1843 version contains, in addition to the aforementioned operation, the division of numbers, this work listed the objective of knowing the principles of this last operation in the cases contained in that text. To this end, a documentary study was carried out looking for information in the past, from the selected text and other writings from the same period
\end{abstract}

${ }^{1}$ suziealbuquerque@hotmail.com

Número Especial - IV Seminário Cearense de História da Matemática

Boletim Cearense de Educação e História da Matemática - Volume 07, Número 20, 323 - 334 (2020)

DOI: $10.30938 /$ bocehm.v7i20.2870 
that revealed how 10th century mathematics practitioners dealt with the division of numbers, especially in the use of Gerbert's abacus. In this sense, a preliminary analysis of the historical context mentioned in three spheres was carried out: the contextual, the historiographic and the epistemological. From this, it was possible to understand that the division of numbers in the use of Gerbert's abacus occurs by subtraction, obtaining the quotient of the idea of how many times the dividend can contain the divisor. In addition, it brings the division by the method of differences, in which the operation is performed dividing by an auxiliary divider that would facilitate the obtaining of the results in the abacus system. Although this is an initial study, it is possible to indicate elements that can collaborate in understanding the operation of the division through its modern algorithm. However, this last aspect will be deepened in future studies.

Keywords: Division of the numbers. Gerbert's abacus. Interface between history and teaching.

\section{Introdução}

A articulação entre a história e o ensino de matemática tem sido tema em número crescente de estudos em nível nacional desde a década de 90, com a criação da Sociedade Brasileira de história da matemática, fomentando oo surgimento de pesquisas na área com foco na educação matemática. Nesse âmbito, pesquisas mais recentes ${ }^{2}$ têm investido na construção de interface ${ }^{3}$ entre as duas áreas mencionadas a priori, partindo do uso de documentos históricos ${ }^{4}$ antigos para buscar recursos a serem inseridos no ensino, sobretudo na formação de professores de matemática.

Saito e Dias (2013), por sua vez, adotam a vertente historiográfica atualizada na construção da referida interface, sendo esta abordagem da história um meio de compreender a matemática do passado sem empregar significados modernos a conceitos antigos, evitando-se anacronismos. Pereira e Saito (2018) consideram esta forma de adentrar no passado como um dos movimentos da construção da interface, importante para a compreensão dos processos de elaboração, transmissão e transformação dos conhecimentos matemáticos.

O outro movimento na interface, denominado de movimento do pensamento (PEREIRA; SAITO, 2018), está relacionado com a formação dos conceitos matemáticos, se dá na articulação entre os conhecimentos do passado e do presente. Entretanto, considerando os aspectos do contexto histórico.

Nessa perspectiva, valeu-se da escolha de um texto histórico, o Traité de Gerbert, na versão de 1843 , que teria sido escrito originalmente por volta do ano de

\footnotetext{
${ }^{2}$ Vide Albuquerque (2019), Alves (2019) e Soares (2019)

${ }^{3}$ Vide Saito e Dias (2013) e Pereira e Saito (2018).

${ }^{4}$ Vide Silva (2018)
} 
Um estudo sobre a operação da divisão dos números contida no Traité de Gerbert na versão de 1843 Suziê Maria de Albuquerque

976. Com base nas informações contidas nesse texto, buscou-se realizar um primeiro movimento que compreende a pesquisa documental, fundamentado no estudo preliminar de três esferas: a contextual, a historiográfica e a epistemológica (ALFONSO-GOLDFARB; FERRAZ; WAISSE, 2013).

Com vista a conhecer os princípios da operação de divisão nos casos contidos no Traité de Gerbert, na versão de 1843, será apresentado a seguir inicialmente um esboço do contexto histórico do período medieval estudado, seguido de uma discussão detalhada sobre os achados no Traité de Gerbert concernente à ideia inicial sobre operação da divisão dos números e o método da divisão por diferença.

\section{O contexto histórico em torno das matemáticas medievais que emerge do Traité de}

\section{Gerbert na versão de 1843 no que diz respeito ao uso do ábaco}

O Traité de Gerbert foi escrito originalmente por volta do ano de 976 da era cristã, no território do reino da França. Neste período o Ocidente Latino, vivenciava um movimento de recuperação dos textos antigos advindos sobretudo do povo grego que estava sobre o domínio árabe (DUBY, 1967). Neste cenário, a Igreja Católica desempenhou papel significativo na aquisição, reprodução e difusão dos conhecimentos contidos nesses textos, sobretudo nos que se referem às artes liberais ${ }^{5}$.

Além disso, o final das invasões bárbaras proporcionou a expansão comercial e a sensação de segurança, sobretudo no transporte das mercadorias, que se estenderam para locais mais distantes, seja por terra, vias fluviais ou marítimas (LE GOFF, 2003). Tais fatores intensificaram a necessidade da realização de cálculos cada vez mais precisos e ágeis, tendo em vista a competitividade no mercado.

Entretanto, o sistema de numeração romano em vigor nessa região não oferecia recursos para a rapidez nos cálculos, tendo em vista que este evidenciava o caráter cardinal, ou seja, considerava as quantidades em si. Essa característica pode ser observada no ábaco romano ${ }^{6}$.

O trabalho com o cálculo do valor de grandes cargas de mercadorias ou medição de distâncias entre os portos de venda tornavam inviável o investimento nesse mecanismo. Havendo a demanda de buscar um sistema de cálculo que otimizasse a

\footnotetext{
${ }^{5}$ Correspondiam ao Trivium (retórica, gramática e lógica) e ao Quadrivium (aritmética, geometria, astronomia e música)

${ }^{6}$ Vide Ibiapina (2014)
}

Número Especial - IV Seminário Cearense de História da Matemática

Boletim Cearense de Educação e História da Matemática - Volume 07, Número 20, 323 - 334 (2020)

DOI: $10.30938 /$ bocehm.v7i20.2870 
Um estudo sobre a operação da divisão dos números contida no Traité de Gerbert na versão de 1843 Suziê Maria de Albuquerque

obtenção do resultado final, implicando diretamente no desenvolvimento da sociedade medieval.

Nesse contexto, Gerbert de Aurilac (946-1003), um religioso beneditino que posteriormente viária a se tornar o Papa Silvester II (999-1003), recebendo instruções nas artes e tendo acesso a escritos antigos gregos, apresenta a seus discípulos regras de cálculo que contaria com a redução do número de fichas a serem utilizadas no ábaco para a representação dos valores.

No sistema proposto pelo referido erudito, foram confeccionadas cerca de mil fichas com a reprodução de nove algarismos para representar todos os número (RICHER, 1845). Apesar de haver indícios históricos da existência desses símbolos e seu uso no ábaco, o próprio Gerbert (1843) não apresenta suas características, nem descreve a estrutura física do instrumento em seu tratado.

Porém, a partir do estudo de tratados do mesmo período foi possível ter acesso à descrição da estrutura física do ábaco em questão por meio de textos como o de Richer, na versão de 1845, que foi um dos discípulos de Gerbert, bem como de Bernelinus de Paris que escreveu outro tratado sobre este ábaco.

Outro achado importante para a compreensão do formato adotado para o uso do ábaco foi um manuscrito catalogado como Abacus Trier, escrito originalmente por volta do ano de 1081, presente a um abade de Trier. Este ábaco teria sido produzido na cidade de Echternach em Luxemburgo (BURNETT, 2010).

A estrutura do instrumento era semelhante a do ábaco romano mostrada por Ifrah (2007), no entanto, possuía alguns elementos a mais que os diferenciavas. A partir de tal esboço foi possível reconstruir o instrumento (FIGURA 1).

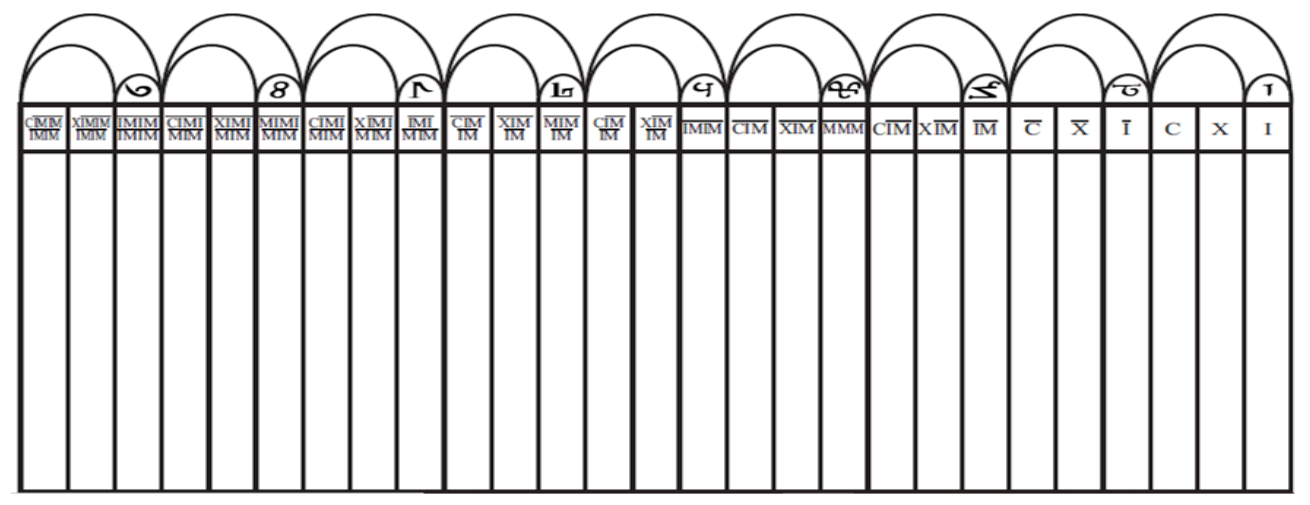

Figura 1 - Reconstrução do ábaco de Echternach Fonte: Albuquerque (2019, p. 48) 
A composição do ábaco é apresentada com um conjunto de vinte e sete colunas verticais, em cuja parte superior são afixadas as inscrições que correspondem ao valor assumido por cada coluna. Os compartimentos são agrupados, totalizando nove arcos de três tipos, um que envolve de três em três colunas, outro que envolve a primeira coluna de cada agrupamento de três e outro arco intermediário que engloba a segunda e a terceira coluna de cada grupo de três.

No interior de cada arco menor está inscrito um símbolo que, segundo Chasles (1843c) era utilizado para representar os números no ábaco. Da direita para a esquerda, correspondem respectivamente aos números 1, 2, 3, 4, 5, 6, 7, 8 e 9. Contam-se nove algarismos com os quais se podia escrever qualquer número, bastava combinar os mesmos entre si, de forma que cada coluna corresponderia a um valor determinado.

O símbolo passava assim a representar um número e não uma quantidade, como ocorre com o uso dos algarismos romanos. Um mesmo algarismo poderia representar quantidades diferenciadas, a depender da posição que este fosse localizado no ábaco. Esse conhecimento preliminar sobre o instrumento conduz na identificação de conhecimentos matemáticos que possam estar sintetizados nessa construção.

Nesse sentido, Albuquerque (2018) discorre sobre os conhecimentos matemáticos que estão sintetizados na estrutura desse ábaco que, segundo as evidências apresentadas, corresponde a um instrumento semelhante ao utilizado por Gerbert (1843) e seus discípulos. Se os conhecimentos antigos incorporados no uso do ábaco forem articulados com os conceitos modernos, percebe-se que as colunas podem ser representadas como potências na base 10 e os arcos serem associados ao uso do ponto e da vírgula atual que colaboram na leitura e identificação das ordens e classes numéricas.

Todavia, anterior a esse movimento do pensamento entre os conhecimentos do passado e do presente, é relevante praticar as regras contidas no texto histórico de maneira que se as matemáticas antigas se tornem compreensíveis.

Assim, vale informar preliminarmente o conteúdo do Traité de Gerbert, que é formado por vinte e cinco casos para a operação da multiplicação e dez capítulos explicativos sobre os procedimentos para a divisão dos números (ALBUQUERQUE; PEREIRA, 2018; ALBUQUERQUE, 2019).

A seguir serão discutidos dois dos capítulos que tratam da divisão, abordando uma ideia inicial que fundamenta a referida operação no período medieval em questão e o manuseio do ábaco para um caso da divisão pelo método das diferenças. Para tanto, 
Um estudo sobre a operação da divisão dos números contida no Traité de Gerbert na versão de 1843 Suziê Maria de Albuquerque

serão descritos os procedimentos metodológicos adotados e os princípios que conduziram o estudo na perspectiva da construção da interface entre história e ensino de matemática.

\section{A operação da divisão dos números a partir das regras de cálculo contidas no}

\section{Traité de Gerbert}

A divisão no ábaco que consta no Traité de Gerbert foi organizada por Chasles (1843a) em dez capítulos que tratam de divisões particulares em ordem crescente de complexidade, conduzindo à compreensão do que seja o resultado da divisão efetuada.

De início, Gerbert (1843, p. 286, tradução nossa) explica o princípio da divisão que permeia os demais capítulos de seu texto, que se intitula de "a divisão de um número de unidades por uma série de unidades, ou um número de dezenas por um número de dezenas, etc."7. De maneira que o divisor e o dividendo pertencem a uma mesma coluna ou ordem do ábaco.

$\mathrm{Na}$ sequência é informado que “[...] para dividir unidades por unidades, ou dezenas por dezenas, ou centenas por centenas, ou milhares por milhares, devemos subtrair um do outro, considerando-os como meros elementos, isto é, de acordo com sua denominação"8 (GERBERT, 1843, p. 286, tradução nossa). Logo, a ideia de divisão parte da subtração do divisor em relação ao dividendo. De acordo com Chasles (1843b) o termo denominação corresponde ao valor absoluto do número.

A divisão que remete à ideia de "quantas dezes o divisor cabe no dividendo", o que revela o conceito de quociente. Apesar de Gerbert (1843) citar de forma explicita esse termo apenas no último capítulo desta parte do seu escrito. Portanto, o dividendo corresponde ao maior número e o divisor ao menor número, afinal o manuseio do ábaco conduz ao uso de números positivos, maiores ou iguais a uma unidade.

No caso exposto (Figura 2), o número 2 pode ser subtraído 4 vezes do número 8 , o que implica que 8 dividido por 2 é igual a 4. De modo semelhante ocorre a divisão de dezenas por dezenas, centenas por centenas e das demais colunas por elas mesmas. No ábaco, tem-se:

\footnotetext{
7 Vide "Division d'un nombre des unités par un nombredes unités, ou d'un nombre des dizaines par un nombredes dizaines, etc." (GERBERT, 1943, p. 286).

${ }^{8}$ Vide "D'après cela, pour diviser des unités par des unités, ou des dizaines par des dizaines, ou des centaines par des centaines, ou des mille par des mille, il faut retrancher les uns des autres, en les considérantcomme de simplesunités, c'est-à-dire suivant leur dénomination” (GERBERT, 1943, p. 286).
} 


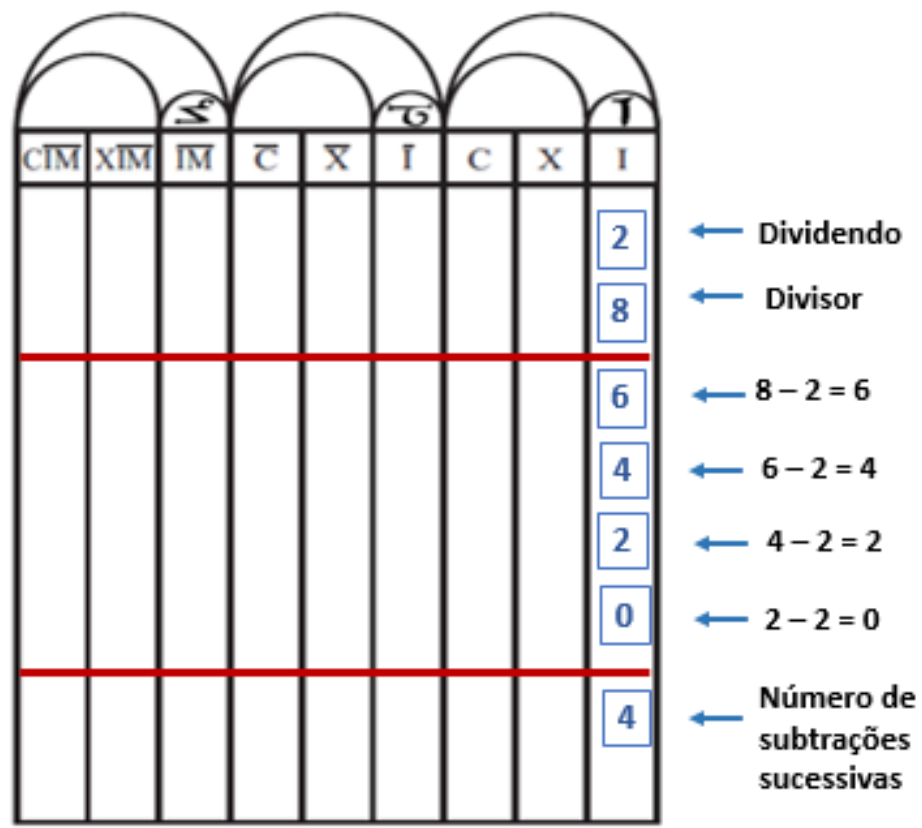

Figura 2 - A divisão no ábaco por meio da subtração ${ }^{9}$ Fonte: Elaborado pela autora (2019)

Este princípio pode ser aplicado nos demais capítulos, mas com o diferencial da utilização de um divisor auxiliar para realizar os cálculos, como orienta Gerbert (1843, p. 286, tradução nossa) ${ }^{10}$ no segundo capítulo do seu tratado, quando discorre sobre a divisão por unidades:

Na divisão dos números do Ábaco, as unidades são para as dezenas, para as centenas e para as milhares, assim como as dezenas para as centenas e para os mil, e as centenas para os mil e dez mil, e os mil para os dez mil e cem mil. De acordo com isso, para dividir dezenas, centenas ou milhares, em conjunto, ou com intervalo por unidades, teremos a diferença do divisor em dez; será multiplicado pela denominação total do dividendo; Se os artigos resultarem, a divisão continuará operando em sua própria denominação e com a diferença já feita. Quanto aos dígitos, eles serão adicionados aos dígitos; e se vier dos artículos, dividiremos, como acima, até que possamos ter apenas dígitos. Se alguém tiver um milhar (para dividendos), os itens (da multiplicação da diferença pela denominação do dividendo) serão colocados

\footnotetext{
${ }^{9}$ Assim como na multiplicação, a disposição dos números no ábaco não foram descritas nessa versão do texto de Gerbert, adotou-se então o padrão detectado em tratados do mesmo período.

${ }^{10}$ Vide "Dans la division des nombres de l'Abacus comme les unités sont aux dizaines, aux centaines et aux mille, ainsi sont les dizaines aux centaines et aux mille, et les centaines aux mille et aux dix-mille, et les mille aux dixmille et aux cent-mille. D'après cela, pour diviser des dizaines, ou des centaines, ou des mille, réunis, ou avec intermission par des unités, on prendra la différence du diviseur à dix; on la multipliera par la dénomination entière du dividende; s'il en résulte des articles, on continuera la division em opérant sur leur propre dénomination et avec la différence déjà posée. Quant aux digits, on les ajoutera aux digits; et s'il en provientdes articles, on divisera, comme ci-dessus,jusqu'à ce qu'on arrive à n'avoir que des digits. Si l'on a des mille (pour dividende) les articles (provenant de la multiplication de la différence par la dénomination du dividende) seront placés dans les mille, et les digits dans les centaines si l'on a des centaines, on placera les articles dans les centaines et les digits dans les dizaines si l'on a des dizaines, on placera les articles dans les dizaines, et les digits dans les unités” (GERBERT, 1843, p. 286).
} 
nos milhares, e os dígitos nas centenas, se houverem centenas, um irá colocar os artigos em centenas e os dígitos nas dezenas se alguém tiver dezenas, um colocará os artigos nas dezenas e os dígitos nas unidades.

Chasles (1843a) considera essa regra lacônica e de difícil entendimento se ele não tivesse acessado outros manuscritos do período para compreender a chamada divisão pelo método da diferença ${ }^{11}$, recebendo esta nomenclatura por recorrer a um divisor auxiliar. Este número com o qual se dividirá é tomado se fixando a diferença dele para dez.

Desta forma, ao se dividir por 8 , tem-se 2 como a diferença de 8 para 10 . A intenção é ao invés de dividir por 8, ser por 10. Este artifício facilitava os cálculos no ábaco, pois as colunas estão dispostas em potências de 10, como explicado anteriormente.

Na figura 3 será exposto o registro do divisor, dividendo e diferença do divisor a serem tomados. A divisão sem diferença, ou sendo tomado apenas o divisor 8, implicaria em sucessivas subtrações do número unidades mais as que sobrarem das 4 dezenas. No entanto, se os números forem considerados como unidade simples, como aconselha Gerbert (1843), não seria possível subtrair 8 de 4, por exemplo.

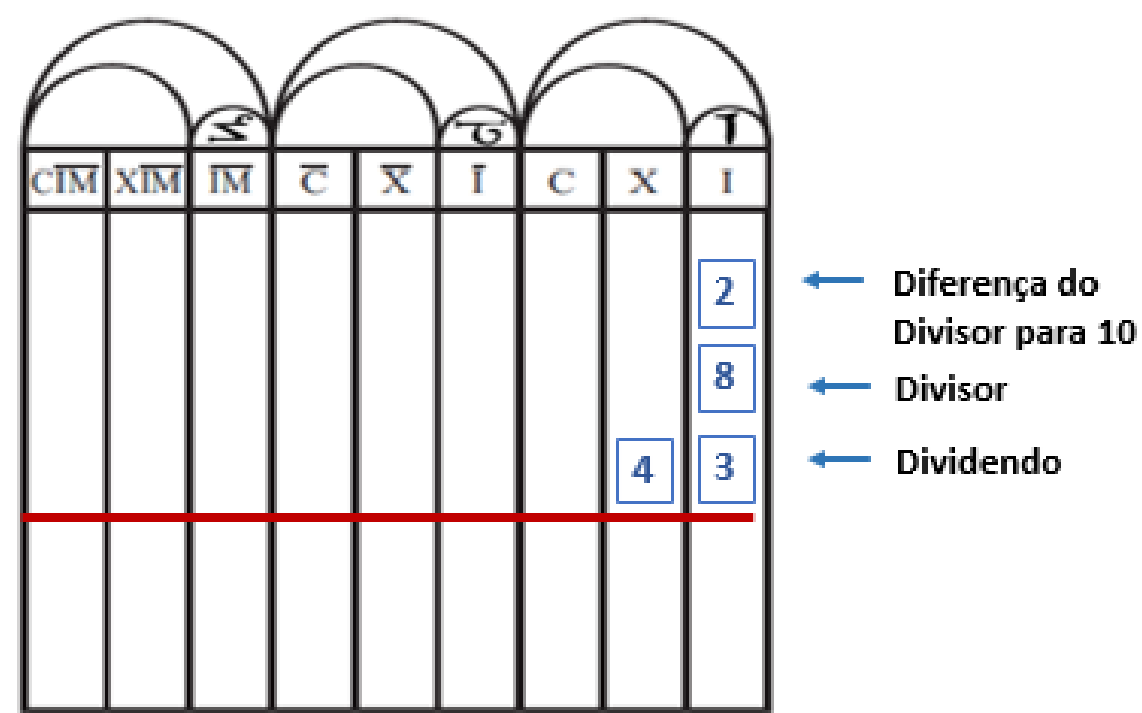

Figura 3 - Início da divisão pelo método das diferenças no ábaco Fonte: Elaborado pelas autoras (2019)

Se forem considerados os números nas colunas em seu valor absoluto, em termos de denominação, pelo método descrito por Gerbert, ao se dividir por 10, a

11 A nomenclatura método da diferença não aparece no texto original, mais foi assim denominada por Chasles (1843b).

Número Especial - IV Seminário Cearense de História da Matemática
Boletim Cearense de Educação e História da Matemática - Volume 07, Número 20, 323 - 334 (2020)

DOI: $10.30938 /$ bocehm.v7i20.2870 
Um estudo sobre a operação da divisão dos números contida no Traité de Gerbert na versão de 1843 Suziê Maria de Albuquerque

divisão tem início na coluna de maior valor posicional. Na coluna das dezenas se divide 4 dezenas por 10, que resulta em 4 unidades (FIGURA 3). Assim, esse dígito será escrito na coluna das unidades. Como a divisão está sendo realizada por um divisor diferente do principal, é necessário corrigir o erro como descrito no tratado, seguindo o procedimento (FIGURA 4):

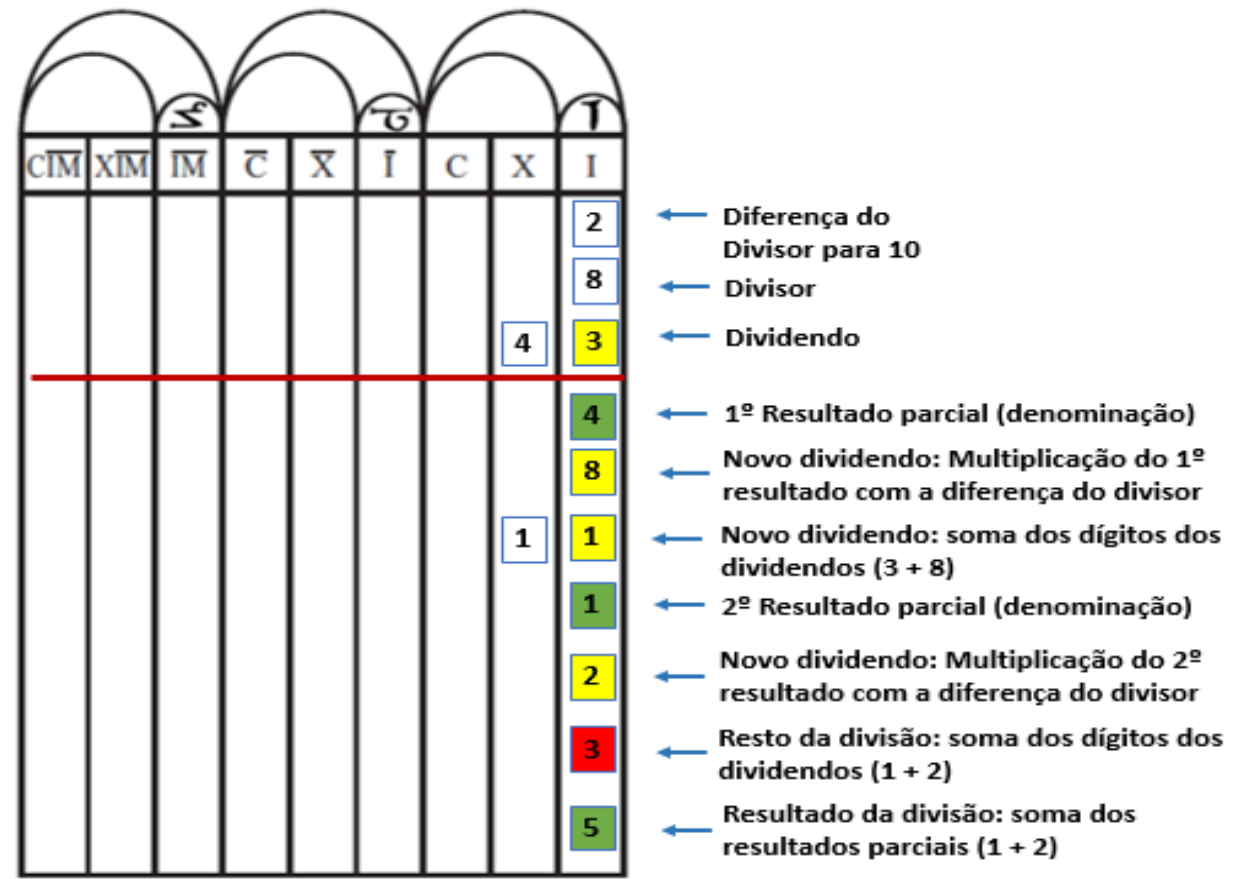

Figura 4 - Procedimento da divisão pelo método da diferença Fonte: Elaborado pelas autoras (2019)

O primeiro resultado parcial é obtido verificando quantas vezes se subtrai 10 de 40. Opera-se então com o artículo do dividendo, reservando o dígito, o número 3. O resultado dessa primeira divisão é o dígito 4 que foi inserido na coluna das unidades. Com o uso do divisor auxiliar, é necessário corrigir o erro, para isso se multiplica o primeiro resultado parcial pela diferença do divisor para 10, determinando um novo dividendo (4.2=8).

Entretanto, 8 não é divisível por 10, sendo necessário somar os dígitos dos dois dividendos que ficaram reservados $(3+8=11)$, tomando-se esse valor como novo divisor, pois possui dígito e artículo. Assim, o segundo resultado é o dígito 1, deixando o artículo 1 do dividendo reservado. O último resultado é multiplicado pela diferença do divisor para $10(1.2=2), 2$ é o novo divisor. 
Porém, 2 é dígito é divisível por 10, ele fica reservado para somar com os demais dígitos que ainda não foram somados $(1+2=3)$. Esta soma corresponde ao resto da divisão. O resultado final é encontrado somando as denominações dos resultados parciais $(4+1=5)$. Dessa maneira, verifica-se que $43=8.5+3$.

Diante da execução da divisão pelo método da diferença, considera-se, de forma genérica, a e b como algarismos do dividendo e d como o divisor. Com o valor das colunas em termos de potência de 10, como ocorreu na explicação da multiplicação, tem-se que cada resultado ou quociente parcial corresponde ao articulo do dividendo multiplicado por $10^{\mathrm{n}-1}$, sendo inserido, portanto, uma linha abaixo, na coluna à direita do artículo dividendo, tendo em vista que o mesmo foi dividido por 10.

Esse resultado parcial $\left(\mathrm{a} .10^{\mathrm{n}-1}\right)$ é multiplicado em seguida por $(10$ - d), correspondendo ao novo divisor. A divisão prossegue até que todos os artículos e dígitos forem divididos. No final, soma-se todos os resultados parciais, o que implica no resultado geral do cálculo.

Além desse caso da divisão, o Traité de Gerbert contém outros específicos que foram listados no capítulo anterior. Entretanto, estes serão discutidos em outras oportunidades, tendo em vista que a ideia de divisão discutida é suficiente para o prosseguimento desta pesquisa.

\section{Algumas considerações}

Os estudos que vem sendo realizados na construção de interface entre a história e o ensino de matemática tem permitido a educadores matemáticos se apropriarem de saberes históricos que podem (re) significar os conhecimentos consolidados em sua formação, implicando na busca por recursos que possam ser incorporados no ensino.

De modo específico quando se discute a divisão dos números de acordo com o Traité de Gerbert, percebe-se que esta pode colaborar com o ensino desta operação adotando procedimento diferenciado do moderno, acarretando na compreensão da ideia principal da divisão.

Entretanto, este estudo preliminar se restringiu ao estudo contextual e histórico, havendo espaço para estudos posteriores que aprofundem os conhecimentos matemáticos mobilizados no manuseio do instrumento para os casos da divisão dos números de modo que sejam elencados os elementos potencialmente didáticos para o ensino de matemática. 
Um estudo sobre a operação da divisão dos números contida no Traité de Gerbert na versão de 1843 Suziê Maria de Albuquerque

\section{Referências}

ALBUQUERQUE, Suziê Maria de. Um estudo sobre a articulação contida no Traité de Gerbert (1843) e o ensino na formação de professores de matemática. Dissertação (Mestrado) - Curso de Mestrado em Ensino de Ciências e Matemática, Instituto Federação de Educação, Ciência e Tecnologia do Ceará, Fortaleza, 2019.

ALBUQUERQUE, Suziê Maria de; PEREIRA, Ana Carolina Costa Pereira. A divisão por unidades pelo método das diferenças: uma proposta de uso do ábaco de Gerbert.

Revista de produção discente em educação matemática. v.7, n.2, 2018. Disponível em: < https://revistas.pucsp.br/pdemat/article/view/39027>. Acesso em 15 de jan. de 2019 às $11 \mathrm{~h} 30 \mathrm{~min}$.

ALFONSO-GOLDFARB, Ana Maria; FERRAZ, Marcia; WAISSE, Silvia. Reflexões sobre a constituição de um corpo documental para a história da ciência: um estudo de caso do brasil colônia e brasil reino. Acervo - Revista do Arquivo Nacional, v. 26, n. 1, jan-jun: Arquivos e história das ciências, n. 1, p. 42-53. Disponível em: <http://hdl.handle.net/20.500.11959/brapci/107915>. Acesso em: 01 jan. 2020.

ALVES, Verusca Batista. Um estudo sobre os conhecimentos matemáticos mobilizados no manuseio do instrumento círculos de proporção de William Oughtred. Dissertação (Mestrado) - Curso de Mestrado em Ensino de Ciências e Matemática, Instituto Federação de Educação, Ciência e Tecnologia do Ceará, Fortaleza, 2019.

BURNETT, Charles. Numerals and Arithmetic in the Middle Ages. Ashgate Publishing Company: Burlington, 2010.

CHASLES, Michael. Analyse et explication du traité de Gerbert. In Académie des sciences (France). Comptes rendus hebdomadaires des séances de l'Académie des sciences / publiés... par MM. les secrétaires. Gauthier-Villars: Paris, 1843a, p. 281 285. Disponível em: <https://gallica.bnf.fr/ark:/12148/bpt6k2976b?rk=21459;2\#>. Acesso em 16 de jan. de 2018 às 18 h 00min.

CHASLES, Michael. Règles de l'Abacus (traduction littérale). In Académie des sciences (France). Comptes rendus hebdomadaires des séances de l'Académie des sciences / publiés... par MM. les secrétaires. Gauthier-Villars: Paris, 1843b, p. 218 246. Disponível em: <https://gallica.bnf.fr/ark:/12148/bpt6k2976b?rk=21459;2\#>. Acesso em 16 de jan. de 2018 às 18h 00min.

CHASLES, Michael. Histoire de l'arithmétique: développement et détails historiques sur divers points du système de l'abacus. In Académie des sciences (France). Comptes rendus hebdomadaires des séances de l'Académie des sciences / publiés... par MM. les secrétaires. Imprimerie de Bachelier: Paris, 1843c, p. 1 - 28. Disponível em: < https://gallica.bnf.fr/ark:/12148/bpt6k62063k?rk=21459;2 >. Acesso em 16 de jan.de 2018 às $18 \mathrm{~h} 30 \mathrm{~min}$. 
Um estudo sobre a operação da divisão dos números contida no Traité de Gerbert na versão de 1843 Suziê Maria de Albuquerque

DUBY, Georges. O ano mil. Lisboa: Edições 70, 1967.

GERBERT. Traité de Gerbert. In: Académie des sciences(France). Comptes rendus hebdomadaires des séances de l'Académie des sciences / publiés... par MM. les secrétaires. Gauthier-Villars: Paris, 1843, p. 281 - 295. Tradução por Michael Chasles. Disponível em: <https://gallica.bnf.fr/ark:/12148/bpt6k2976b?rk=21459;2\#>. Acesso em 16 de jan. de 2018 às $18 \mathrm{~h} 00 \mathrm{~min}$.

IBIAPINA, Wilter Freitas. Uso Pedagógico do ábaco romano para o ensino do algoritmo de multiplicação. 2014. 190 f. Dissertação (Mestrado) - Curso de Programa de Pós-graduação em Ensino de Ciências Naturais e Matemática, Universidade Federal do Rio Grande do Norte, Natal, 2014.

PEREIRA, Ana Carolina Costa; SAITO, Fumikazu. Os instrumentos matemáticos na interface entre história e ensino de matemática: compreendendo o cenário nacional nos últimos 10 anos. In: SEMINÁRIO CEARENSE DE HISTÓRIA DA MATEMÁTICA, 3, 2018, Fortaleza. Anais. Fortaleza: Eduece, 2018, p. 1 - 12.

RICHER. Histoire de son temps. Paris: L'imprimerie de Crapelet, 1845. 4 v. Traduzido por J. Guadet.

SAITO, Fumikazu; DIAS, Marisa. Interface entre história da matemática e ensino: Uma atividade desenvolvida com base num documento do século XVI. Ciência \& Educação, São Paulo, v. 19, n. 1, p.89-111, 2013.

SOARES, Francisco Wagner Oliveira. Sobre os conhecimentos geométricos Incorporados na construção e no uso do instrumento jacente no plano de Pedro Nunes (1502-1578) na formação do professor de matemática. Dissertação (Mestrado) - Curso de Mestrado em Ensino de Ciências e Matemática, Instituto Federação de Educação, Ciência e Tecnologia do Ceará, Fortaleza, 2019. 